\title{
Higher Order Low Pass FIR Digital Differentiators
}

\author{
Simranjot Singh \\ Research Associate \\ ECED, Thapar University \\ India
}

\author{
Kulbir Singh \\ Associate Professor \\ ECED, Thapar University \\ India
}

\begin{abstract}
In this paper design of non-recursive higher order low pass digital differentiators satisfying given specifications is investigated. The concept of low pass differentiation is further generalized to higher order differentiators. A formula is derived using Fourier integral to compute impulse response coefficients of the differentiator. The equation is then used to design first order differentiators and results are compared with Salesnick's technique. The proposed FIR low pass differentiator has improvement in transition width and flexibility to choose cutoff frequency. The same technique has been demonstrated for second order design according to provided specifications. This method is used in the design of second order low pass differentiator for QRS detection in ECG. It is shown that the proposed implementation has low hardware and software complexity as compared to existing second derivative based techniques of QRS detection, giving advantage in optimization of current real time ECG systems.
\end{abstract}

\section{General Terms}

Signal Processing, Biomedical Signal Processing, Higher Order Differentiator Design, Digital Differentiator, QRS Complex Detection, Fourier Integral

\section{Keywords}

FIR Filter, Higher order differentiator, Low pass differentiator, QRS complex detection, Fourier Integral

\section{INTRODUCTION}

This paper describes the design of linear phase finite impulse response (FIR) higher order low pass digital differentiators. An expression is introduced, to calculate impulse response coefficients, by using Fourier series design method. Then window filter design technique is employed along with Kaiser Window to design filters.

Low pass digital differentiators are used to avoid unwanted amplification of noise, as in case of full band ones [1]. Salesnick provided recursive formula to design first order FIR low pass differentiator's impulse response coefficients in [1]. Alaoui described the recursive design procedure of low pass differentiators in [3]. Alaoui's approach proved to better in transition width as compared to Salesnick's method. The question of higher order case of low pass differentiators' design becomes significant as they suffer from this limitation even more, because of their exponentially increasing gain. Corresponding higher order case is investigated in this paper. Higher order differentiation is an important signal processing technique, found in many applications such as biomedical signal processing and calculations of geometric moments [2]. Low pass differentiator can be implemented by a single filter or with cascading of differentiator and low pass filter [3]. The frequency response of higher order ideal full band differentiator is given by

$$
H(\omega)=(j \omega)^{v} \quad|\omega|<\pi
$$

where $v$ is a natural number for differentiators.

The higher order low pass differentiator is characterized by ideal frequency response as

$$
\begin{array}{rlrl}
H(\omega) & =(j \omega)^{v} & & |\omega|<\omega_{c} \\
& =0 & \omega_{c}<|\omega|<\pi
\end{array}
$$

where $\omega_{s}=2 \pi$ is the sampling frequency in radians per second. Here $\omega_{c}$ is cutoff frequency such that $\omega_{c}=k \pi, k$ is the parameter to control cutoff frequency in designing practical low pass differentiators.

Accurate QRS complex detection is important in $\mathrm{R}$ peak detection and R-R interval extraction from ECG. An improvement in existing second derivative QRS complex detection methods could be achieved by low pass differentiators.

The paper is developed as follows. In the first section, the formula to calculate filter coefficients of higher order low pass FIR digital differentiator is derived using Fourier integral. Then window method of FIR filter design and optimization algorithm used in design examples are discussed briefly. In second section, first order low pass differentiator is designed for different given specifications, the results are compared with present approaches. Then the same technique is explained for second order case design examples. Finally, an improved second order derivative based algorithm, for QRS complex detection, is proposed as an application of the discussed method

\section{HIGHER ORDER DIGITAL DIFFERENTIATOR}

All Impulse response coefficients of the differentiator is calculated by using Fourier series technique of filter design [4], [5], [6], expressed as

$$
h(n)=\int_{-\pi}^{\pi} H(\omega) \exp \{j \omega n\} d \omega
$$

Using (1) and from [7], the integral can be written as

$$
\begin{aligned}
h(n) & =\left[\frac{\exp \{j \omega n\}}{n}\left\{(j \omega)^{v}-\frac{v}{n}(j \omega)^{v-1}+\ldots \ldots\right\}\right]_{-k \pi}^{k \pi} \\
& =\left[\frac{\exp \{j \omega n\}}{n} \sum_{q=0}^{v} \frac{v !(j \omega)^{v-q}(-1)^{q}}{(v-k) ! n^{q}}\right]_{-k \pi}^{k \pi}
\end{aligned}
$$

Let $l=v-q$, then 


$$
\begin{aligned}
h(n) & =\left[\frac{\exp \{j \omega n\} v !(-1)^{v}}{n^{v+1}} \sum_{l=0}^{v} \frac{(-j \omega n)^{l}}{l !}\right]_{-k \pi}^{k \pi} \\
& =\left[\frac{1}{n}\left(\frac{-1}{n}\right)^{v} \exp \{j \omega n\} v ! \sum_{l=0}^{v} \frac{(-j \omega n)^{l}}{l !}\right]_{-k \pi}^{k \pi} \\
& =\left[\frac{1}{n}\left(\frac{-1}{n}\right)^{v} \Gamma(v+1,-j \omega n)\right]_{-k \pi}^{k \pi} \\
& =\frac{1}{n}\left(\frac{-1}{n}\right)^{v}[\Gamma(v+1,-j k \pi n)-\Gamma(v+1, j k \pi n)]
\end{aligned}
$$

So the discrete time system is obtained as

$$
H(z)=\sum_{n=-N_{1}}^{N_{1}} h(n) z^{-n}
$$

The amplitude response of ideal digital differentiator is

$$
H_{i}(\omega)=|\omega|
$$

Then amplitude response of the designed differentiator is given by

$H_{d}(\omega)=\left|\sum_{n=-N_{1}}^{N_{1}} h(n) \exp \{-j \omega n\}\right|$

The error in amplitude response of designed digital differentiator is

$$
E(\omega)=H_{d}(\omega)-H_{i}(\omega)
$$

The impulse response of a first order digital differentiator is shown in Fig. 1.

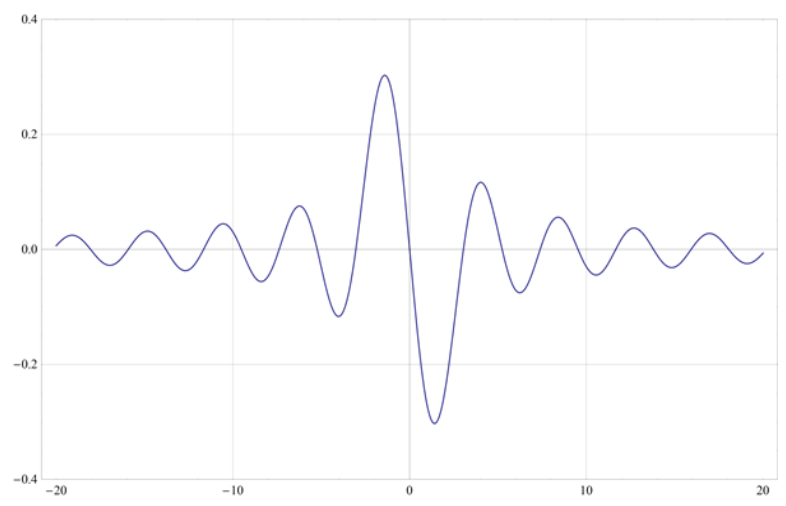

Fig. 1. Impulse response $k=0.35, v=1$

In order to obtain finite length of impulse response, the Fourier series is truncated such that

$h(n)=0$ for $|n|>(N-1) / 2$

where $N$ is length of the filter and an odd integer.

Due to truncation of Fourier series, Gibbs oscillations are introduced in the frequency response of the differentiator.
These oscillations' amplitude can be reduced by the use of window functions [4], [6]. This preconditioning result in transfer function of the filter given by

$H(z)=\sum_{n=-\infty}^{\infty}\{w(n) h(n)\} z^{-n}$

In this paper Kaiser window is used as it provides extra degree of freedom by providing shaping parameter $\alpha$. This feature of Kaiser Window helps in the designing process of digital differentiator. Kaiser window function is given by following expression

$$
\begin{aligned}
w(n) & =I_{0}(\beta) / I_{0}(\alpha) & \text { for } \quad|n| \leq N_{1} \\
& =0 & \text { otherwise }
\end{aligned}
$$

Parameter $\beta$ is given by

$$
\beta=\alpha \sqrt{1-\left(\frac{n}{N_{1}}\right)^{2}}
$$

where $N_{1}=(N-1) / 2$ and $I_{0}(x)$ is zeroth order Bessel function of first kind.

A parameter $L(\alpha, N)$ can be defined as maximum error in passband of the practical digital differentiator, such that

$L(\alpha, N)=\max |E(\omega)|$ for

$0 \leq \omega \leq \omega_{c}$

(8)

In order to achieve minimum of $L(\alpha, N)$ with respect to $\alpha$ ,this optimum value can be calculated from an optimization procedure consisting of a combination of golden section search and successive parabolic interpolation as given in [8] and [9]. This algorithm requires three parameters, an initial interval of values of $\alpha$ and tolerance. The working of algorithm is reduction of the interval of uncertainty on each iteration by the factor 0.618 until it is less than the tolerance. Therefore it is better than bisection based counterparts. This method is faster computationally and is used to find optimum value of $\alpha$ in design examples of this paper.

\section{FIRST ORDER LOW PASS DIFFERENTIATOR}

The design process can be categorized into three parts, such that the differentiator is designed for given specifications.

\subsection{Differentiator design with minimum passband error for a given value of $N$}

Please Optimum value of $\alpha$ is found using the optimization algorithm for provided $N$. Once we know $N, \alpha$ and $v$ the differentiator can be designed by using (6). Some results of the algorithm are given in Table I rows and columns show progression of optimization procedure for $N=29, v=1$ and $\omega_{c}=0.35 \pi$.

Table I. Progress chart of optimization algorithm

\begin{tabular}{|l|l|l|l|}
\hline$N$ & $\alpha^{*}$ & $L\left(\alpha^{*}, N\right)$ & $\begin{array}{l}\text { Number of } \\
\text { Iterations }\end{array}$ \\
\hline 25 & 4.44 & 0.0051 & 10 \\
\hline
\end{tabular}




\begin{tabular}{|l|l|l|l|}
\hline 31 & 5.6 & 0.0016 & 10 \\
\hline 39 & 7.16 & 0.00034 & 11 \\
\hline
\end{tabular}

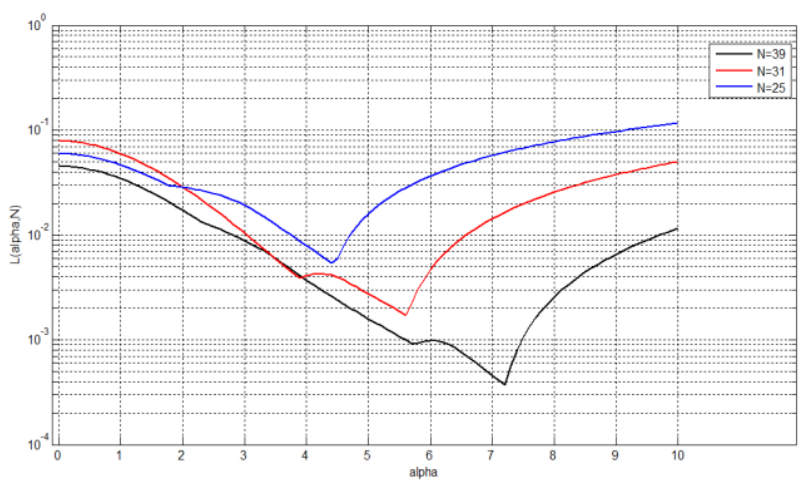

Fig.2. $\mathbf{L}(\alpha, \mathbf{N})$ curve for $\omega_{c}=\mathbf{0 . 3 5} \pi$ and $\mathbf{v}=1$.

\subsection{Differentiator design with minimum $N$ for a provided in band error $\delta$}

An important observation from Fig. 2 and Table I, is linearity in plot as $N$ is increased. This linearity in plots of $\ln \left(e / e_{1}\right)$ against $N-N_{1}$ for different values of $N_{1}$ [10] can be exploited, to calculate value of $N$ only by two minimizations. As it can be seen in Fig. 2, global minima points almost lie on a straight line.

Minimum value of $N$ which achieves the specified inband error can be calculated by procedure given in [10]. In this method if

$e=L\left(\alpha^{*}, N\right)$ and $e_{1}=L\left(\alpha_{1}^{*}, N_{1}\right)$

Where $\alpha_{1}{ }^{*}$ and $\alpha^{*}$ are global minima point for $N_{1}$ and $N$ respectively of designed digital differentiator for a particular normalized cutoff frequency $\omega_{c}$, then

$N=N_{1}+\frac{\ln \left(e / e_{1}\right)}{\ln \left(e_{2} / e_{1}\right)}\left(N_{2}-N_{1}\right)$

This formula calculates $N$ such that it satisfies user requirement given by

$L\left(\alpha^{*}, N\right) \leq \delta$

Here $\delta$ is maximum acceptable error in passband of low pass digital differentiator.

For example, if $\omega_{c}=0.35$ then by using optimization procedure described in previous section, $\alpha_{1}{ }^{*}$ and $\alpha_{2}{ }^{*}$ are computed for $N_{1}=25$ and $N_{2}=29$ with $\delta$ taken as 0.0005 . Then following results are obtained

$N_{1}=25 \alpha_{1}^{*}=4.44 e_{1}=0.0051$

$N_{2}=31 \alpha_{2}^{*}=5.6 e_{2}=0.0016$
It yields $N=39$ and $e$ comes out less than 0.0004 .

\subsection{Differentiator design with given in band accuracy and $N$ to obtain minimum transition width}

In many applications error of $2 \%$ is acceptable in passband of low pass differentiator [3], therefore transition width can be optimized under this constraint. Salesnick's and Alaoui's approaches are compared with the Fourier series technique as in Fig 3 and Fig. 4. It can be seen that designed differentiator's response is better in terms of transition region and sharp cut off characteristics. These features are important for suppression of high frequency noise. The overshoot,present in the amplitude response after reaching $\omega_{c}$ , decreases as $\alpha$ increases but so does error in passband. A relatively higher value of $\alpha$ is selected for minimum overshoot under $2 \%$ constraint as shown in Fig. 3. In this case $\alpha$ is taken 6.6

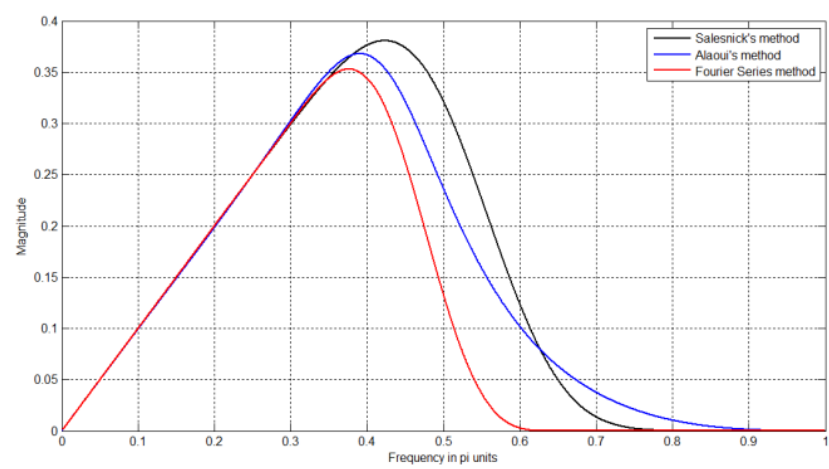

Fig. 3. Magnitude response comparison for $\omega_{c}=\mathbf{0 . 3 5}$

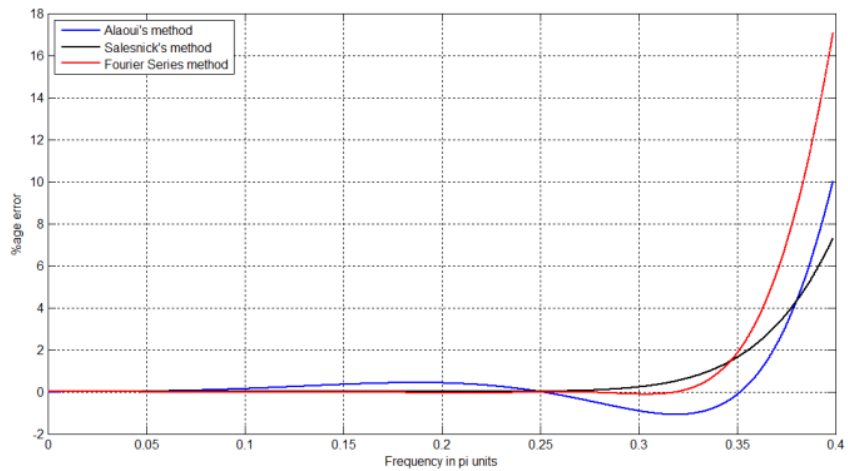

Fig. 4. Error comparison for $\omega_{c}=\mathbf{0 . 3 5}$ in passband

\section{SECOND ORDER LOW PASS DIFFERENTIATOR}

To obtain good performance at zero normalized frequency a constraint on coefficients is imposed [11] so that

$$
\sum_{n=-N_{1}}^{N_{1}} h(n)=0
$$

Same options as in first order case can be taken here as well. 


\subsection{Differentiator design with minimum passband error for a given value of $N$.}

Optimum $\alpha$ is found using golden section optimization and progress chart of some of them is provided in Table II.

Table II Progress chart for $\omega_{c}=\mathbf{0 . 3 5} \pi$ and $\mathrm{N}=\mathbf{2 9}$

\begin{tabular}{|l|l|l|l|}
\hline$N$ & $\alpha^{*}$ & $L\left(\alpha^{*}, N\right)$ & $\begin{array}{l}\text { Number of } \\
\text { Iterations }\end{array}$ \\
\hline 25 & 4.42 & 0.00757 & 10 \\
\hline 31 & 5.55 & 0.00245 & 11 \\
\hline 39 & 7.11 & 0.00047 & 10 \\
\hline
\end{tabular}

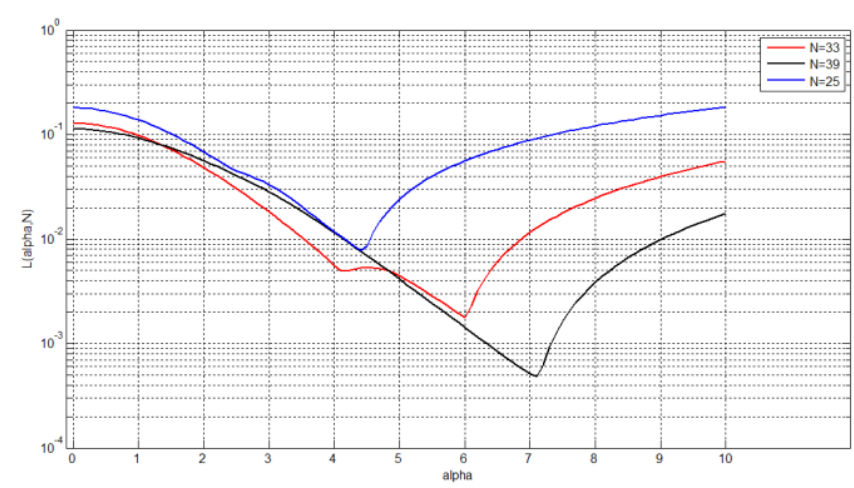

Fig.5. $\mathbf{L}(\alpha, \mathbf{N})$ curve for $\mathrm{k}=\mathbf{0 . 3 5}$ and $\mathrm{v}=2$

\subsection{Differentiator design with minimum $N$ for a provided in band error $\delta$}

Linearity in plots of $\ln \left(e / e_{1}\right)$ against $N-N_{1}$ for different values of $N_{1}$ is present in second order differentiator also, as can be seen in Fig. 5. Therefore the procedure to design differentiator with minimum $N$ could be applied in this case also for given $\delta$. It can be easily verified by checking Table II and taking $\delta$ as 0.0005 .

\subsection{Differentiator design with given in band accuracy and $N$ to obtain minimum transition width.}

As discussed in last section amplitude response can be optimized to attain given accuracy in passband. This feature is due to the fact that $\alpha$ can be varied to any fractional value as opposed to Salesnick's approach. The Fig. 6 shows the amplitude response of differentiator of impulse response length 29 and normalized cut off frequency $0.55 \pi$ with applied constraint of $2 \% . \alpha$ has value of 5.5. Percentage error plotted in Fig. 7 of the designed differentiator shows in band error is less than specified constraint.

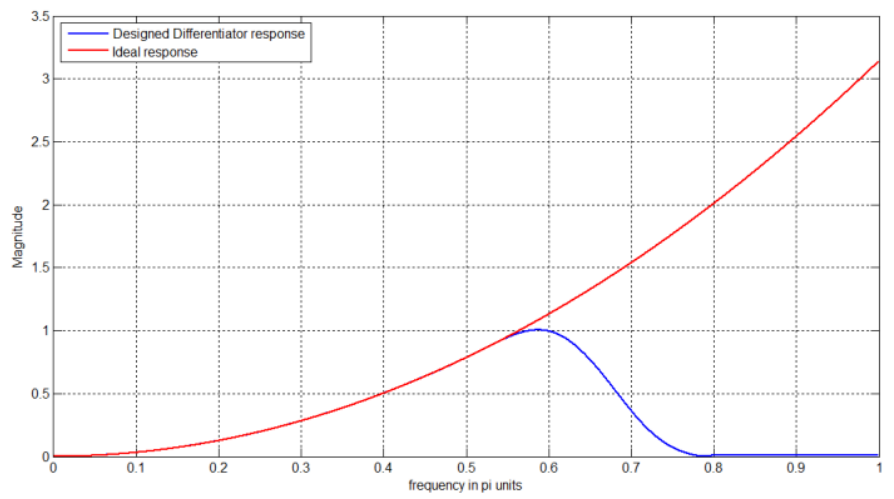

Fig.6. Frequency response for $\mathrm{v}=2, \omega_{c}=\mathbf{0 . 5 5}$ and $\mathrm{N}=\mathbf{2 9}$

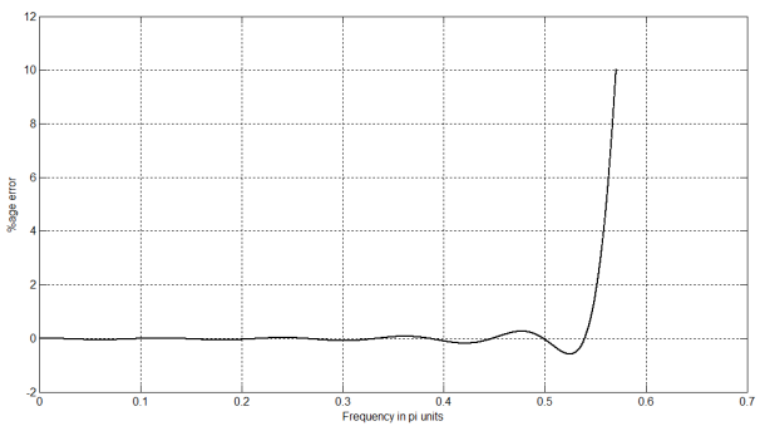

Fig.7. Percentage error for $\mathrm{v}=2, \omega_{c}=\mathbf{0 . 5 5}$ and $\mathrm{N}=29$

Fig. 8 illustrates application of same technique for different values of $\omega_{c}$.

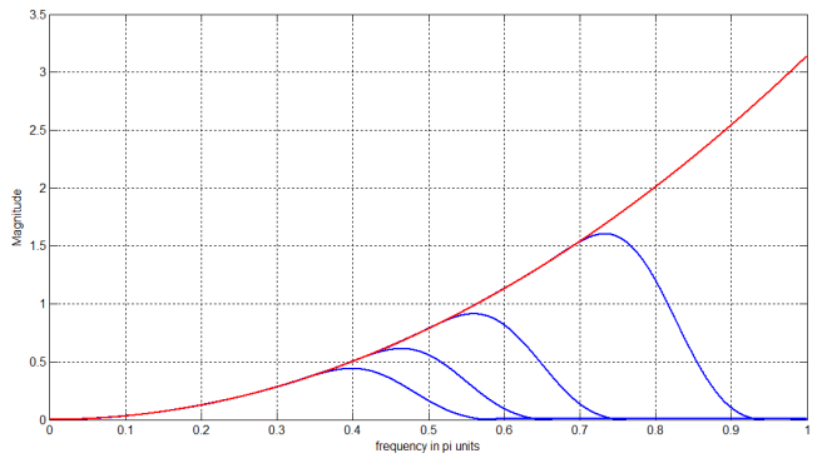

Fig. 8. Magnitude response for $\mathrm{v}=\mathbf{2}, \mathrm{N}=\mathbf{2 9}$ and $\omega_{c}=\mathbf{0 . 3 5 \pi}$, $0.42 \pi, 0.52 \pi, 0.7 \pi$

\section{QRS COMPLEX DETECTION}

In this section an application of higher order FIR low pass differentiator is demonstrated. Algorithms based on derivatives are suitable for real time applications [12]. It illustrates the use of these filters in biomedical signal processing, where low frequency components of signal are important. QRS complex detection is needed in beat and beatto-beat interval information in an electrocardiogram (ECG) recording [12]. It is crucial to accurately detect QRS, as it contains P and T wave, as well as noises [13] and artifacts as described here. An ECG with such distortions is shown in Fig. 11 (a). 
(i) Power line interference consisting of $60 \mathrm{~Hz}$ frequency and harmonics.

(ii) Electromyographic interference, which is random high frequency noise.

(iii) Baseline drift due to respiration or abrupt shift in baseline.

Second derivative based method, as described in [12], is used here. The structure of the technique is shown in Fig. 9. A

Kaiser window based FIR band pass filter is employed to pass the QRS complex frequencies such that 8 to $20 \mathrm{~Hz}$ passband. First differentiator is a center differentiation stage and second differentiator is forward differentiation stage. Zero crossings are formed in the locations of $\mathrm{R}$ waves after first differentiation process, therefore another differentiation is required to form peaks at $\mathrm{R}$ waves. This introduces delay in the sequence.

The adaptive threshold is computed from root mean squared (RMS) value of a data segment. In the end, if a peak is detected then a search back algorithm is used, it based on 200 $\mathrm{ms}$ physiological refractory period before another QRS complex can occur. Therefore another peak present in $200 \mathrm{~ms}$ window is stored for further analysis.

Digital differentiators are high pass filters and their low pass case can be considered as a band pass filter, especially at higher order differentiation. The structure of this technique is modified such that band pass filter is no longer used and there is also no need of second stage of differentiation. Therefore the first three stages of the structure are highly simplified due to second order low pass differentiator as given in Fig. 10.

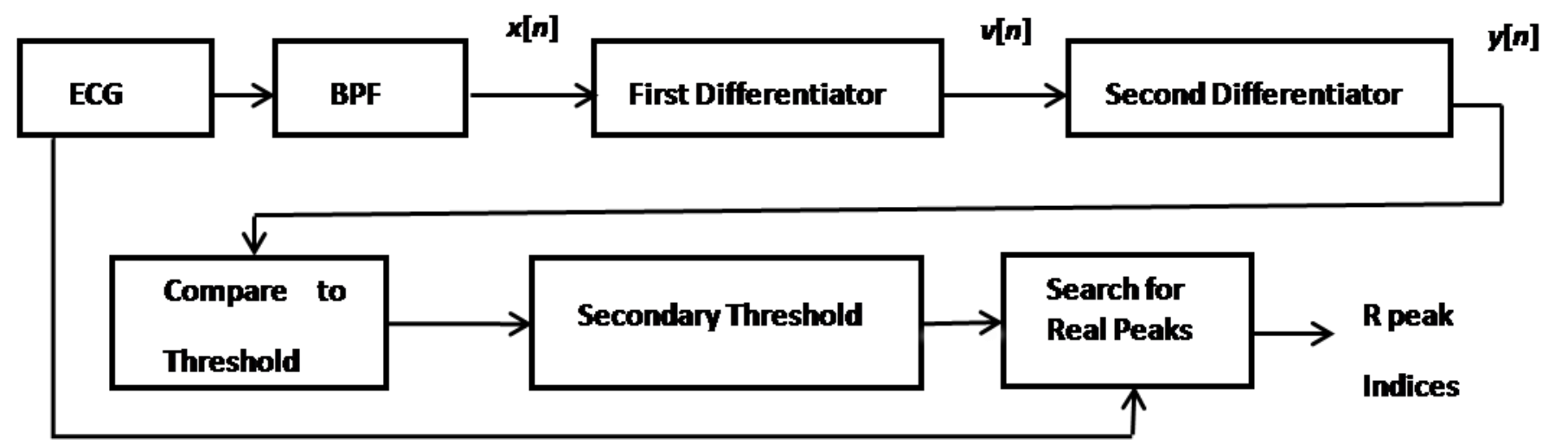

Fig. 9. Second derivative method (the first technique) structure [12]

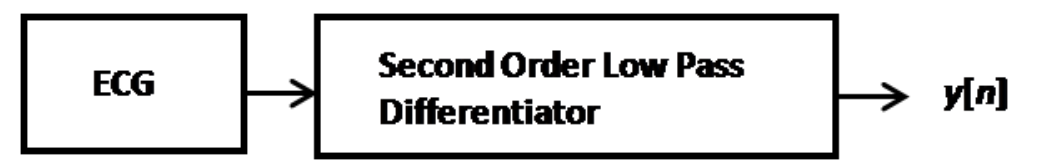

Fig. 10. The proposed low pass differentiator method (the second technique) structure

This technique was applied on MIT/BIH arrhythmia database [14]. These ECG recordings have been sampled at $360 \mathrm{~Hz}$ with 11 bit resolution over the range of $10 \mathrm{mV}$. Record 108 is used in this paper to illuminate the proposed algorithm's performance. This recording has rare long $\mathrm{P}$ waves and high noise contents [15], it has reversed polarity QRS complexes. The second order FIR differentiator's impulse response length is 35 and window parameter is taken 6.33 (from optimization algorithm). The number of coefficients in bandpass filter is also 35. The resultant output, $y[n]$, from both the arrangements is plotted in Fig. 11.

The second derivative method produces peaks with more gradual transition from zero voltage, as given in Fig. 11 (b). The proposed algorithm exhibits sharp outstanding peaks in the location of R peaks, as illustrated in Fig. 11 (c). It should also be noted that number of coefficients in bandpass filter, of first technique, is the same as number of coefficients in second order low pass differentiator of the second technique. First method requires two stages of differentiation after the filter, however this method embeds the three operations in the filter of same length. Therefore number of operations required by the proposed method to detect QRS complex are reduced. This feature is crucial in real time implementation of QRS detection of patient's ECG and Holter tape monitoring [16] also. Although this paper presents QRS complex detection application, the proposed approach can be employed in second derivative based biomedical signal processing.

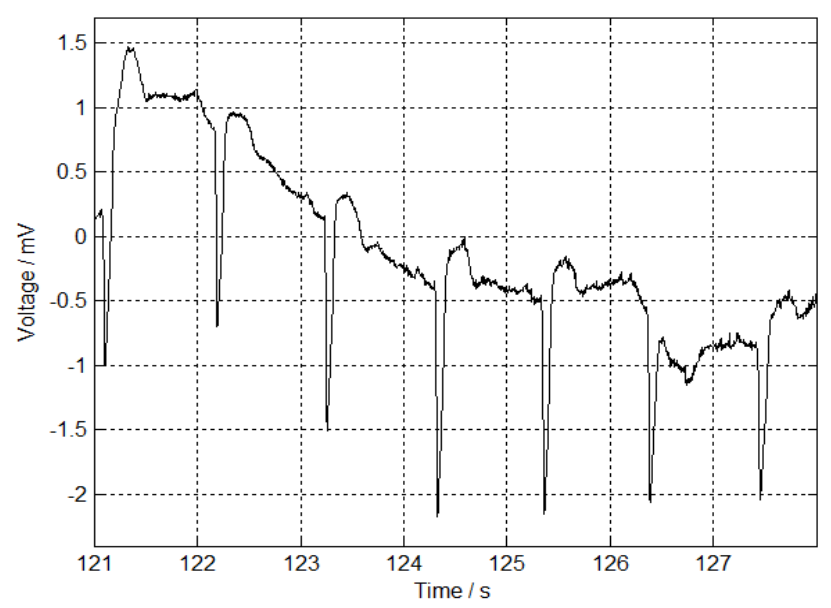

(a) 


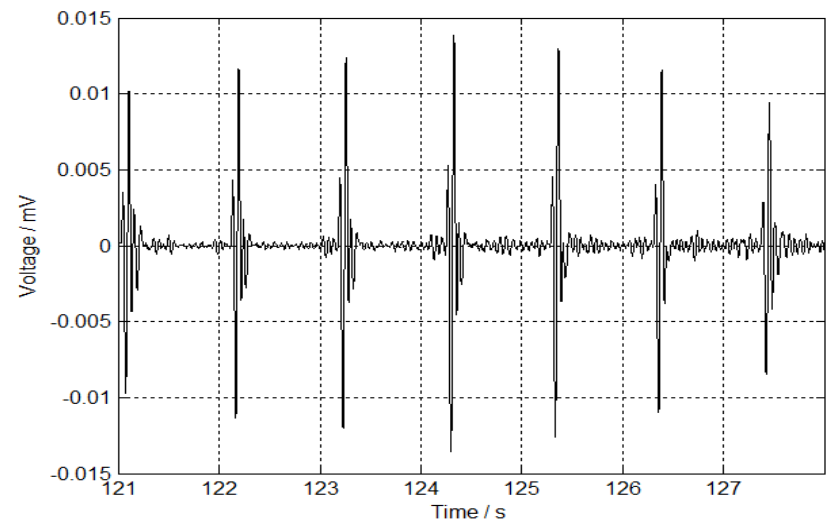

(b)

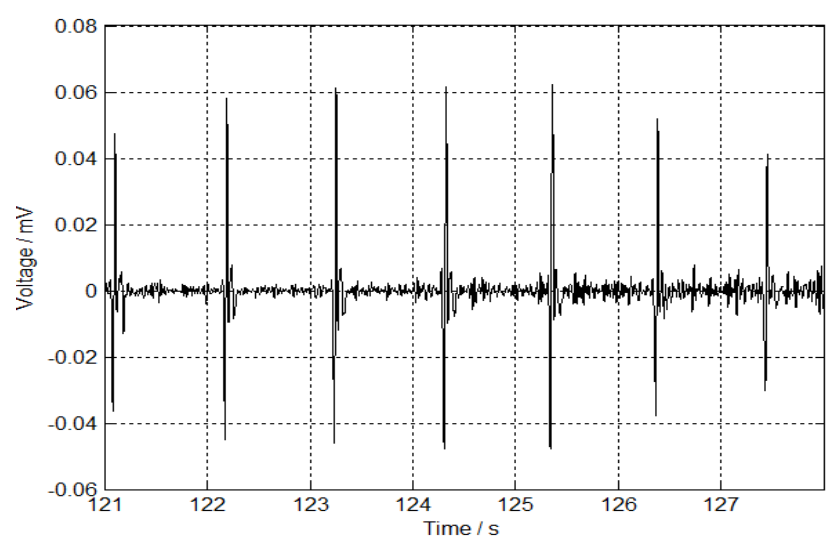

(c)

Fig. 11. (a) ECG signal from Record 108; (b)y[n] output from first algorithm; (c) y[n] output from proposed algorithm

\section{CONCLUSIONS}

This paper describes the design of higher order case of FIR low pass digital differentiators. It is based on Fourier series method for design of filters along with Kaiser Window function

The derived expression is used to design first order differentiator with varying normalized cutoff frequency using Kaiser Window. It provides an easy way to change cutoff frequency without altering the sampling frequency, reducing aliasing risk in the system. The frequency response of designed differentiator is compared with Salesnick's [1] and Alaoui's [3] approaches. The proposed differentiator is having shorter transition regions and less overshoot in frequency response. Flexibility in design process is observed due to possibility of window shaping parameter to fractions. Second order differentiators are produced and error analysis is illustrated by graphs. Different design options available to user due to window shaping parameter are discussed separately.

The formula is employed to obtain second order FIR low pass differentiator for QRS detection in ECG. The proposed algorithm enables us to detect $\mathrm{R}$ peaks even in presence of noise and baseline wanders, as demonstrated by using MIT/BIH ECG recording. This technique also has low complexity as compared to second derivative counterparts, which makes it suitable for real time processing of ECG waveform. This method could well be used in second derivative based biomedical signal processing at a lower computational cost.

\section{REFERENCES}

[1] I. Salesnick, Maximally flat lowpass digital differentiators, IEEE Trans. Circuits Syst. II, vol. 49 (2002), no. 3, pp. 219-223.

[2] S.C. Dutta Roy, B. Kumar, Handbook of Statistics, Vol. 10, Elsevier Science Publishers, Amsterdam, pp.159-205, 1993.

[3] M. A. Al-Alaoui, Linear phase low-pass IIR digital differentiators, IEEE Trans. Signal Process. (2007), vol. 55, no. 2, pp. 691-706.

[4] A. Antoniou, Design of digital differentiators satisfying prescribed specifications, IEEE Proc. E. Comput. Digital Tech., vol. 127(1980).

[5] J. G. Proakis and D. G. Manolakis, Digital Signal Processing. Englewood Cliffs, Prentice-Hall, 1996.

[6] A.V. Oppenheim and R.W. Schafer, Discrete-Time Signal Processing, Prentice-Hall, Englewood Cliffs, 1999.

[7] P. Bois, Table of Infinite Integrals, Dover Publications, NY, 1962.

[8] G. E. Forsythe, M. A. Malcolm, C. B. Moler, Computer Methods for Mathematical Computations, Prentice-Hall, 1976.

[9] P.B. Richard, Algorithms for Minimization without Derivatives, Prentice-Hall, Englewood Cliffs, New Jersey, 1973.

[10] A. Antoniou, C. Charalambous, Improved design method for Kaiser differentiators and comparison with equiripple method, IEEE Proc. E. Comput. Digital Tech. (1981) vol. 128.

[11] H. Zhao, G. Qiu, L. Yao, J. Yu, Design of fractional order digital FIR differentiators using frequency response approximation, Proc. 2005 Int. Conf. Communications, Circuits and Systems. (2005) pp. 1318-1321.

[12] N.M. Arzeno, Z.D. Deng, C.S. Poon, Analysis of first derivative based QRS detection algorithms, IEEE transactions on Biomedical Engg. 55(2008) 478-484.

[13] G.M. Friesen, T.C. Jannett, M.A.Jadallah, S.L. Yates, S.R. Quint, H.T. Nagle, A comparison of the noise sensitivity of nine QRS detection algorithms. IEEE Trans. Biomed. Eng. BME-37 (1990). 85-97.

[14] A. L. Goldberger et al., PhysioBank, PhysioToolkit, and PhysioNet:Components of a new research resource for complex physiologic signals, Circulation, vol. 101 (2000), no. 3, pp. 215-220.

[15] A. Ghaffari, H. Golbayani, M. Ghasemi, A new mathematical based QRS detector using continuous wavelet transform, Computers and Electrical Engineering 34 (2008) 81-91

[16] P.S. Hamilton, W.J. Tompkins, Quantitative investigation of QRS detection rules using the MIT/BIH arrhythmia database. IEEE Trans. Biomed. Eng. BME-33 (1986). 1157-65 